\title{
PARALLELIZING COMPUTER VISION ALGORITHMS ON ACCELERATION TECHNOLOGIES: A SIFT CASE STUDY
}

\author{
Miaoqing Huang, Chenggang Lai \\ Department of Computer Science and Computer Engineering \\ University of Arkansas \\ Email: \{mqhuang, cl004\}@uark.edu
}

\begin{abstract}
Computer vision algorithms, such as scale-invariant feature transform (SIFT), are used in many important applications, e.g., autonomous vehicle and computer-human interaction. They are typically computation intensive and require a long processing time on traditional single-core processors. In this work, we present the methodologies to parallelize the SIFT algorithm on various acceleration technologies and multicore processors, such as field-programmable gate arrays (FPGAs), graphics processing units (GPUs), and Intel Many Integrated Core (MIC) Architecture. The results show that all acceleration technologies can significantly improve the performance compared with the single-thread implementation on an Intel Core i7 processor. In particular the Nvidia Telsa K20 GPU is capable of providing a $10 \times$ speedup. Furthermore, it is found that the performance of the Intel MIC coprocessor is at the same range as the multicore CPU processor, while the optimal implementation of SIFT does not necessarily use all the computing resources on these two platforms.
\end{abstract}

Index Terms - image processing, computer vision, SIFT, parallelization, acceleration.

\section{INTRODUCTION}

SIFT is an algorithm in computer vision to detect and describe local features in images [1]. The whole SIFT process is divided into 8 stages as shown in Table 1. Each of the 8 stages has its own degree of intrinsic parallelism. Among the 8 stages, we find that the last 2 stages are particularly heavy. Therefore, they are parallelized on various technologies for acceleration. In reality, these two stages are almost inseparable and are implemented as one stage, which is represented as $O+K$ afterwards. The Convolution stage performs a convolution operation on the image and suits well on GPU and FPGA.

This work is supported in part by National Science Foundation under grant CNS-1219062 and by National Aeronautics and Space Administration under grant 12-2012EPSCoR-0026. Any opinions, findings, and conclusions or recommendations expressed in this material are those of the authors and do not necessarily reflect the views of the NSF and the NASA.
Table 1. The 8 stages in SIFT and their parallelization on various technologies.

\begin{tabular}{c||c|c|c|c}
\hline \multicolumn{1}{c||}{} & \multicolumn{4}{c}{ Acceleration Technology } \\
\hline & FPGA & GPU & Multicore CPU & Intel MIC \\
\hline \hline Down Sample & & & & \\
\hline Up Sample & & & & \\
\hline Convolution & $\checkmark$ & $\checkmark$ & & $\checkmark$ \\
\hline DoG & & $\checkmark$ & & \\
\hline Find Key & & & & $\checkmark$ \\
\hline Refine Key & & & & $\checkmark$ \\
\hline $\begin{array}{c}\text { Octave Gradient* } \\
\text { Key Description* } \\
\text { Generation }\end{array}$ & $\checkmark$ & $\checkmark$ & $\checkmark$ & \\
\hline
\end{tabular}

${ }^{*}$ Combined as a single $\mathrm{O}+\mathrm{K}$ stage in real implementation.

The Difference-of-Gaussian $(D O G)$ stage is also good candidate on GPU because of the high memory bandwidth on the GPU card and its embarrassing parallelism.

SIFT has been accelerated on many technologies. The work in [2-4] presented several hardware architecture for SIFT on FPGA or ASIC devices. However, they only dealt with quite small images. Several work [5-7] implemented image processing algorithms such as face recognition and SIFT on mobile GPUs, e.g., Nvidia Tegra [8], Adreno [9], and PowerVR SGX [10]. These GPUs are integrated on the same system-on-chip with the CPUs. Their computing capability is quite limited compared with those standalone GPUs such as the one used in this work. For example, the image size in [7] is $320 \times 240$, which is way below the typical resolution of cameras equipped on today's mobile systems. In this work, we carry out experiments on images of the typical sizes captured by most digital cameras.

We first downloaded the C++ implementation of SIFT algorithm from http://www.vlfeat.org/ vedaldi/code/siftpp.html. This implementation is a single-thread version. We then convert the $\mathrm{C}++$ implementation to $\mathrm{C}$ implementation. We further use various parallel techniques to implement multiple stages on various acceleration technologies, including FPGA, GPU, multicore CPU and Intel MIC, in Section 2. In Sec- 


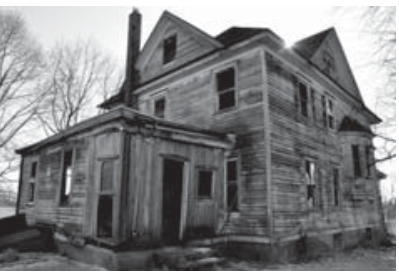

(a) The source image $(4,288 \times 2,848)$.

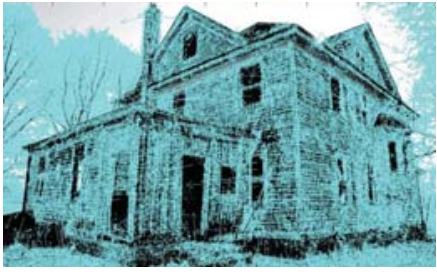

(b) The image augmented with key points.
Fig. 1. Apply the SIFT algorithm on a grayscale image.

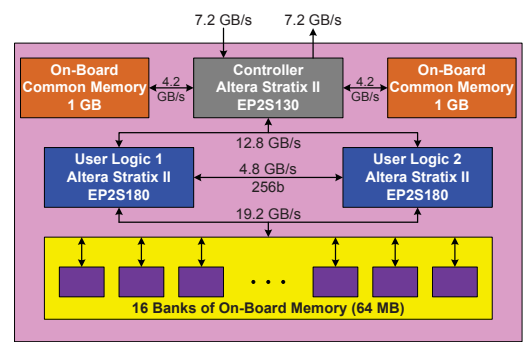

Fig. 2. The MAP reconfigurable processor on SRC-7 computer [13].

tion 3 we carry out a scalability study using images of varying resolutions. Finally, we conclude this work in Section 4.

\section{PARALLELIZING SIFT ON ACCELERATION TECHNOLOGIES}

In this section, we discuss the parallelization details on various processors. For all implementations, we use one workstation or a single node of a computer cluster. We have three implementations as follows.

- Sequential implementation on CPU. The implementation is a single-thread application running on a CPU processor.

- Parallel implementation on multicore CPU or MIC. We use OpenMP [11] to parallelize multiple stages of SIFT on multicore CPU. On Intel MIC processor, we use MPI programming model [12] to distribute workload among the cores on a single processor.

- Hybrid implementation. The sequential part of a program runs on the CPU and the parallel part runs on the accelerator.

We use the grayscale image shown in Fig. 1. The size of the image is $4,288 \times 2,848$, the typical image resolution used by most digital cameras.

\subsection{FPGA}

Field-programmable gate arrays (FPGAs) are integrated circuits designed to be configured by a customer or a designer after manufacturing. Modern FPGAs have the capacity to accommodate complicated algorithms and can be used to speedup a variety of applications [14]. There are a couple of vendors that provide complete reconfigurable computer systems in which FPGAs are used as accelerators, such as SRC and Convey. There are also many vendors that provide FPGA boards to be used for application implementation, such as GiDEL.

The platform used in this work is an SRC-7 reconfigurable computer, which contains 4 GB main memory, 2 Intel Xeon EM64T 3.0GHz CPUs, and one MAP reconfigurable processor. The MAP processor contains two Altera FPGAs as shown in Fig. 2. The user can implement an application using either one or two FPGAs. Both devices can access the 16 banks of on-board memory (OBM) in parallel. There are two approaches to developing applications on the SRC-7 system. The first approach is to use hardware description languages, such as Verilog and VHDL, which requires hardware design skills. The second approach is to use high level language, i.e., MAP-C [13] on SRC-7. An application written in MAP$\mathrm{C}$ will be compiled into Verilog description, which will be then implemented in hardware. Two mechanisms are leveraged to take the advantages of hardware. (1) The compiler will attempt to pipeline the for loops if there is no data dependency between iterations. (2) The programmer can use pragmas to define parallel sections so that they can execute simultaneously.

Three stages are moved to FPGA for computations in the hybrid implementation as shown in Table 1. In the Convolution stage, which is implemented on one FPGA device, we use one OBM bank for the source image, a second OBM bank for the kernel, and a third bank for the result image. We restructure the data layout so that all the for loops can be implemented as fully pipelined hardware modules. For the last two stages, Octave Gradient and Key Description Generation, they are implemented together in a hardware circuit that occupies two FPGAs. There are 4 parallel sections so that 4 key descriptions can be generated in parallel. For the Convolution stage, hardware implementation can reduce the computation time by $50 \%$, i.e., $29.44 \mathrm{~s} \rightarrow 15.36 \mathrm{~s}$. For the last two stages, the performance improvement is more than 10 times, i.e., $170.75 \mathrm{~s} \rightarrow 16.47 \mathrm{~s}$. Overall, the hybrid implementation is able to provide a $6 \times$ speedup compared with the pure software single-thread implementation on the host CPU, i.e., $202.74 \mathrm{~s} \rightarrow 34.25 \mathrm{~s}$.

\subsection{GPU}

GPU architecture has been evolving for many years. Taking the GPUs developed by Nvidia as examples, it has gone through many generations, such as G80 $\rightarrow$ GT200 $\rightarrow$ Fermi $\rightarrow$ Kepler. Nvidia's latest Kepler GPU architecture contains 15 streaming multiprocessors (SMXes), each of which consists of 192 single-precision cores and 64 double-precision cores, as shown in Fig. 3. GPUs are widely used as accelerators 


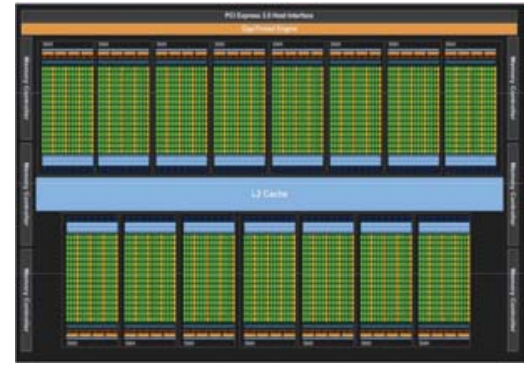

(a) Kepler architecture.

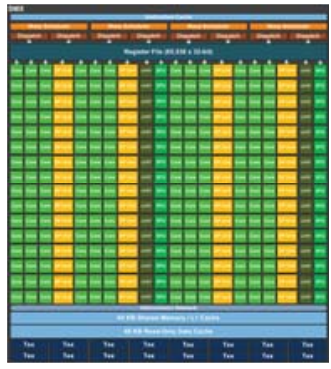

(b) SMX architecture.
Fig. 3. Nvidia's Kepler GPU architecture [15].

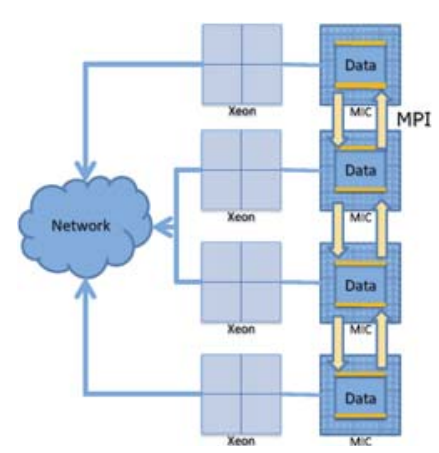

(a) Native mode.

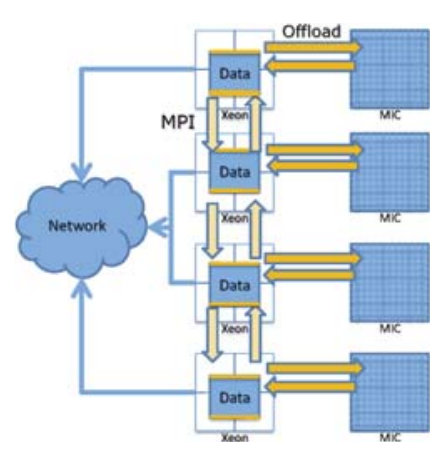

(b) Offload mode.
Fig. 4. Two different approaches to implementing parallelism on MIC based cluster [16].

in high-performance computer clusters as well as individual workstations. The main control of an application typically runs on a host CPU, which allocates the computation to one or more client GPUs. The typical way to implement an application on GPU is to use CUDA [17] or OpenCL [18]. Although these two languages have different flavors, the philosophy for achieving massive parallelism is similar. Basically, the user needs to create a grid of thread blocks, each of which will be scheduled to execute on one SMX during the runtime. In order to keep all cores busy, the number of thread blocks and the number of threads in a thread block are typically much larger than the number of SMXes and the number of cores in each SMX, respectively.

The workstation used in this work contains $16 \mathrm{~GB}$ main memory, an Intel Core i7-3820 3.6GHz CPU and an Nvidia Tesla K20. The K20 GPU contains 13 SMXes and 5 GB memory. We implement both the single-thread CPU version and the hybrid version on the Linux system. The GPU part is implemented using CUDA 5.5. For the hybrid implementation, there are 4 parallel stages shown in Table 1 . Three GPU kernel functions are generated to implemented the Con-

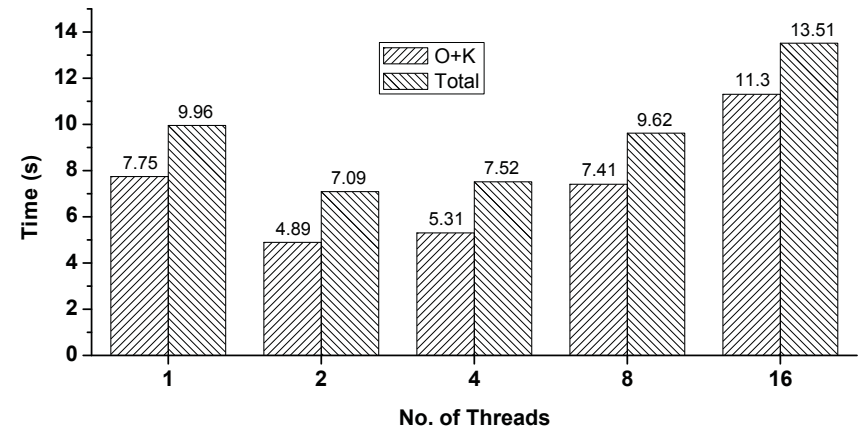

(a) Performance comparison of various parallel SIFT implementations on the 8-core Xeon processor.

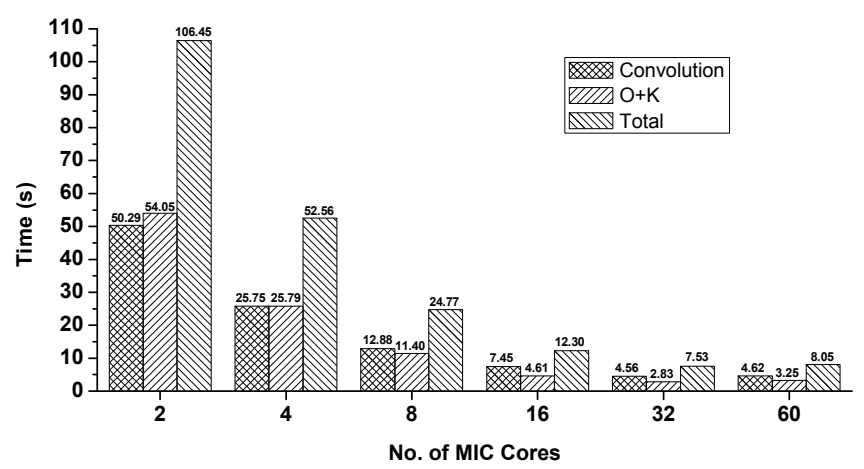

(b) Performance comparison of various parallel SIFT implementations on the Intel MIC coprocessor..

Fig. 5. Tradeoff between performance and parallelism on multicore CPU and Intel MIC.

volution, $D o G$, and $O+K$ stages, respectively. It is found that the $D o G$ stage takes very marginal time even in the sequential implementation. Therefore we focus on the other two kernels. For both kernels, the number of threads in a thread block is 256 . The Core i7 processor has a much higher performance than the Xeon processor used in the SRC-7 system. The single-thread CPU implementation can finish the Convolution and $O+K$ stages in 7.86s and $17.69 \mathrm{~s}$, respectively. Correspondingly, the K20 GPU takes just 1.01s and 1.40s, respectively, for these two stages. Overall, the hybrid implementation is $8.6 \times$ faster than the CPU implementation, i.e., $26.17 \mathrm{~s} \rightarrow 3.03 \mathrm{~s}$.

\subsection{Multicore CPU and Intel MIC}

Intel Many Integrated Core (MIC) Architecture is a manycore processor architecture. The first commercially available Intel coprocessor based on MIC architecture is Xeon Phi, which contains up to 61 scalar processing cores with vector processing units. All cores are integrated using a high-speed bi-directional ring bus. Fig. 4 shows two approaches to paral- 


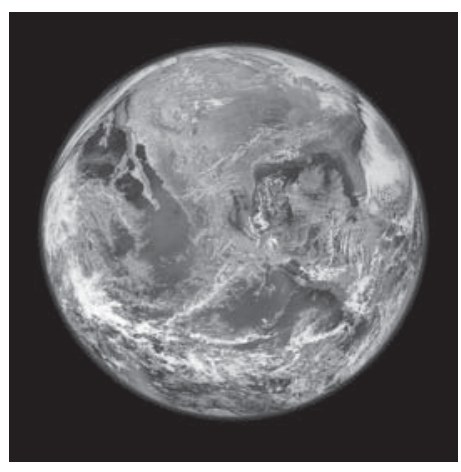

Fig. 6. The spaceborne image of the Earth.

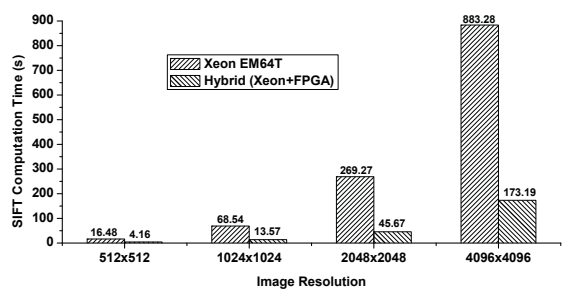

(a) Two implementations on the SRC-7 system.

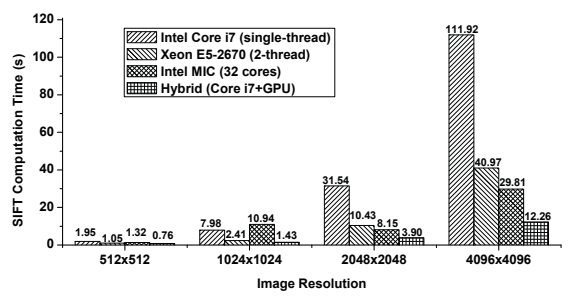

(b) Four implementations on modern CPUs, GPUs, and Intel MIC.

Fig. 7. The performance scalability of various SIFT implementations on images of varying resolutions ( $8 \mathrm{MIC}$ cores are used to process the images of $512 \times 512$ and $1,024 \times 1,024)$.

lelizing applications on computer clusters equipped with MIC processors. The first approach, as shown in Fig. 4(a), is to let each MIC core directly host one MPI process. In this way, the 60 cores on the Xeon Phi 5110P are treated as 60 independent processors while sharing the $8 \mathrm{~GB}$ on-board memory. The second approach is to treat the MIC processors as clients to the host CPUs. As shown in Fig. 4(b), the MPI processes will be hosted by CPUs, which will offload the computation to the MIC processors. Multithreading programming models such as OpenMP can be used to allocate many cores for data processing. We carry out the experiments on a single node of the Beacon computer cluster at the National Institute for Computational Sciences (NICS), University of Tennessee. Each node contains 2 Intel 8-core Xeon E5-2670 2.6GHz processors, 4 Intel Xeon Phi (MIC) coprocessors 5110P, and 256 GB main memory.

We use OpenMP to parallelize the SIFT on the Xeon processor. For the Convolution stage, when we try to parallelize the for loops, it is found that multiple-thread implementation actually takes longer time than the single-thread version due to the complicated memory access pattern. Therefore, only the $O+K$ stage is parallelized on the 8-core Xeon processor. Further, it is found that the higher degree of parallelism does not necessarily bring the better performance. Fig. 5(a) shows that the 2-thread OpenMP implementation of the $O+K$ stage achieves the best performance. When more threads are added, the performance actually becomes worse.

On the Intel MIC coprocessor, it is found that offload mode programming model does not work well for the SIFT algorithm. Therefore, we apply the MPI to implement the native mode in which only MIC cores are used. When using MPI programming model, the data are distributed to all participating cores evenly. Therefore, both Convolution and $O+K$ stages are parallelized. The processing times of all other stages are very marginal. The tradeoff between the performance and the number cores is also observed on the MIC coprocessor. As shown in Fig. 5(b), the 32-core implementation achieves the best performance while performing SIFT on the image shown in Fig. 1(a).

\section{SCALABILITY STUDY}

We further carry out a scalability study of various SIFT implementations on images of varying resolutions. The source image is shown in Fig. 6, which has 4 different resolutions.

On the SRC-7 system, which bears relatively old technologies, the hybrid implementation consistently outperforms the single-thread software implementation on the Xeon EM64T for 4 6 times, as shown in Fig. 7(a). Further, we can find that the performance of the hybrid implementation involving FPGA actually is in the same range of the singlethread implementation on the Intel Core i7 processor. If we use a newer FPGA device and have a better hardware design, it is very positive that the FPGA implementation can outperform modern CPUs while using much less power. By looking at the performance comparison in Fig. 7(b), we can find that the hybrid implementation with GPU clearly establishes its advantage, particularly for large images. In the meantime, both multicore CPU and Intel MIC have very close performances.

\section{CONCLUSIONS}

In this work, we parallelize the SIFT algorithm on multiple accelerators and multicore processors, including FPGA, GPU, Intel MIC, and Intel Xeon processor. Different parallel techniques and languages, such as hardware design, CUDA, OpenMP and MPI, are applied on various technologies. The experiment results clearly demonstrate the advantages for using accelerators and multicore CPUs to improve the performance of computation intensive computer vision algorithms. Among these technologies, GPU can provide the best performance. While Intel MIC and multicore CPUs are very promising, the optimal parallel implementations may not use all the resources on these technologies. 


\section{REFERENCES}

[1] David G. Lowe, "Distinctive image features from scaleinvariant keypoints," International Journal of Computer Vision, vol. 60, no. 2, pp. 91-110, Nov. 2004.

[2] Donghyun Kim, Kwanho Kim, Joo-Young Kim, Seungjin Lee, and Hoi-Jun Yoo, "An 81.6 GOPS object recognition processor based on NoC and visual image processing memory," in Proc. IEEE Custom Integrated Circuits Conference (CICC), Sept. 2007, pp. 443-446.

[3] Vanderlei Bonato, Eduardo Marques, and George A. Constantinides, "A parallel hardware architecture for scale and rotation invariant feature detection," IEEE Trans. Circuits Syst. Video Technol., vol. 18, no. 12, pp. 1703-1712, Dec. 2008.

[4] Lifan Yao, Hao Feng, Yiqun Zhu, Zhiguo Jiang, Danpei Zhao, and Wenquan Feng, "An architecture of optimised SIFT feature detection for an FPGA implementation of an image matcher," in Proc. International Conference on Field-Programmable Technology (FPT), Dec. 2009, pp. 30-37.

[5] Yi-Chu Wang, Bryan Donyanavard, and Kwang-Ting Cheng, "Energy-aware real-time face recognition system on mobile CPU-GPU platform," in Proc. 11th European conference on Trends and Topics in Computer Vision - Volume Part II, Sept. 2010, pp. 411-422.

[6] Kwang-Ting Cheng and Yi-Chu Wang, "Using mobile GPU for general-purpose computing - a case study of face recognition on smartphones," in Proc. 2011 International Symposium on VLSI Design, Automation and Test (VLSI-DAT), Apr. 2011, pp. 1-4.

[7] Blaine Rister, Guohui Wang, Michael Wu, and Joseph R. Cavallaro, "A fast and efficient SIFT detector using the mobile GPU," in Proc. 2013 IEEE International Conference on Acoustics, Speech and Signal Processing (ICASSP), May 2013, pp. 2674-2678.

[8] NVIDIA Corporation, "Variable SMP (4-PLUS-1 ${ }^{\mathrm{TM}}$ )," White paper V1.3, 2011, Available online on http: //www.nvidia.com.

[9] Qualcomm. https://developer.qualcomm. com/discover/chipsets-and-modems/ adreno-gpu.

[10] Imagination Technologies. http://www. imgtec. com/powervr/powervr-graphics.asp.

[11] http://openmp.org.

[12] William Gropp, Ewing Lusk, and Anthony Skjellum, Using MPI: Portable Parallel Programming with the Message Passing Interface (2nd ed), The MIT Press, Cambridge, MA, Nov. 1999.

[13] SRC Computers, LLC, SRC-7 Carte v3.3 C Programming Environment Guide (SRC-023-02), Jan. 2010.

[14] Tarek El-Ghazawi, Esam El-Araby, Miaoqing Huang, Kris Gaj, Volodymyr Kindratenko, and Duncan Buell, "The promise of high-performance reconfigurable computing," IEEE Computer, vol. 41, no. 2, pp. 78-85, Feb. 2008.

[15] NVIDIA Corporation, NVIDIA's next generation CUDA compute architecture: Kepler GK110, 2012.

[16] http://www.jics.tennessee.edu/aace/beacon.

[17] NVIDIA Corporation, NVIDIA CUDA C Programming Guide 5.5, July 2013.

[18] Khronos OpenCL Working Group, OpenCL 2.0 Specification, Khronos Group, Nov. 2013. 